\title{
El debate sobre la evaluación en carreras artísticas
}

\author{
LUCIA GINOCCHIO CASTRO* \\ Instituto de Docencia Universitaria, Pontificia Universidad Católica del Perú \\ Recibido el 10-11-2016; primera evaluación el 06-06-2017; \\ segunda evauación el 25-07-2017; aceptado el 16-08-2017
}

\section{RESUMEN}

En este ensayo se presenta el debate sobre la evaluación en carreras artísticas en el contexto de educación superior. Se exponen los retos y desafíos de la evaluación en las artes y se argumenta la necesidad de esclarecer sus objetivos. Se discuten algunas propuestas que tratan de conciliar el debate. Dichas propuestas son analizadas en relación a su aplicación a favor de la mejora de los procesos de evaluación para la formación universitaria. Finalmente, desde este análisis, se ofrecen recomendaciones para la mejora de los procesos de evaluación en el nivel superior. Dada la reciente fundación de la Facultad de Artes Escénicas de la Pontificia Universidad Católica del Perú (PUCP), y considerando la larga tradición de formación en artes plásticas en la PUCP, se considera este como un tema de gran interés para la pedagogía de las artes y la evaluación en general.

Palabras clave: evaluación, educación superior, pedagogía del arte, carreras artísticas, artes plásticas.

\section{Debate about asessment practices in artistic disciplines}

\section{Abstract}

This essay presents the debate about assessment practices in artistic disciplines in the context of higher education. It exposes the challenges of assessment in the arts and discusses the necessity to clarify its objectives. It also presents some assessment proposals that try to reconcile the debate. These proposals are analysed in relation to their applicability to improve the assessment processes in higher education. Finally, departing form this analysis, it offers recommendations

Licenciada en Psicología con mención en Psicología Educacional por la PUCP y bachiller en Artes Escénicas con mención en Danza por la misma universidad. Actualmente, es coordinadora académica del Área de Investigación del Instituto de Docencia Universitaria, miembro del grupo de investigación en Psicología, Cultura y Género del Departamento de Psicología de la PUCP y se desempeńa como docente de la Facultad de Artes Escénicas y como asistente de docencia en la Especialidad de Psicología de la PUCP. Contacto: lucia.ginocchio@pucp.pe 
to enhance evaluation processes in higher education. Given the recent foundation of the Performing Arts Faculty of PUCP, and considering PUCP's long tradition in fine arts disciplines, this topic is considered of great interest for the pedagogy of the arts and assessment in general.

Keywords: assessment, higher education, artistic pedagogies, artistic careers, visual arts.

\section{Debate sobre a avaliaçáo em cursos de artes}

\section{Resumo}

Neste ensaio se apresenta o debate sobre a avaliação em cursos de artes no contexto do ensino superior. Expóem-se os desafios da avaliação nas artes e se argumenta a necessidade de esclarecer seus objetivos. Discutem-se algumas das propostas que tentam conciliar o debate. Estas propostas são analizadas em relação com sua aplicação em favor da melhora dos procesos de avaliação para a formação universitária. Finalmente, a partir desta análise, oferecem-se recomendaçóes para a melhora dos processos de avaliaçáo no nível superior. Levando em conta a recente fundação do Curso de Artes Cênicas da PUCP, e considerando a longa tradição da formação em artes plásticas na PUCP, considera-se este um quesito de grande interesse para a pedagogia das artes e da avaliação em geral.

Palavras-chave: avaliação, ensino superior, educação artística, carreiras de arte, arte.

\section{INTRODUCCIón}

"Instead of assessment systems serving learning and creating in our discipline, learning and creating in our discipline are being asked to serve assessment systems". (Hope \& Wait, 2013)

La evaluación en carreras artísticas es un campo que no queda exento de dificultades en su planteamiento y ejecución. En la cita que encabeza este texto, Hope y Wait (2013) hacen referencia a uno de los principales problemas a los que se enfrentan educadores artísticos, ¿sirve la evaluación para capturar el verdadero aprendizaje y creatividad de las carreras artísticas, o los procesos de evaluación están, por el contrario, limitándolos?

El debate sobre la evaluación en contraposición con la creatividad se encuentra presente. Como lo expresa Gruber (2008), entre los educadores artísticos se presenta el dilema ideológico de dar notas a los estudiantes, ya que los mismos ven la evaluación en contraposición a la experiencia creativa. En Estados Unidos los marcos de rendición de cuentas juegan, en la actualidad, un papel dominante en la enseñanza y aprendizaje de la educación 
artística, principalmente, en la educación básica. En este contexto, la evaluación es percibida como una barrera para la promoción de la creatividad en los estudiantes (DeLuca, 2010).

A este debate, se suman complejidades adicionales del campo de la evaluación. Por un lado, los docentes que evalúan los procesos de formación artística de sus estudiantes acarrean una larga tradición de enseñanza y métodos de evaluación pocos medidos y validados y prácticas que se han perpetuado ausentes de sustento. Además, la educación artística ha venido planeando e implementado programas de calidad en conocimientos y juicio artístico aunque sin recursos para explicar adecuadamente cómo es que este proceso de aprendizaje artístico se logra (McRorie, 1997).

Por otro lado, el campo de la educación artística es, en si mismo, difícil de objetivar. Las prácticas ejemplares en educación artística no son fáciles de caracterizar, la mejor educación en artes no es fácil de definir, simple de medir, ni se puede encajonar (Carpenter, 2006). El aprendizaje del arte ha sido considerado demasiado visual, afectivo y cualitativo como para poder ser medido objetivamente (Wilson, 1968) y además, es por naturaleza de carácter ambiguo (Hope \& Wait, 2013) y dinámico (Boughton, 1997). Estas características plantean retos importantes en el intento de una medición y clasificación de sus productos.

A diferencia de la ciencia, que busca respuestas universales y univocas, el arte trata usualmente de generar un set de respuestas múltiples concebidas por un creador individual con objetivos particulares para un trabajo o performance específico (Hope \& Wait, 2013). El arte, además, es un proceso de creación a partir de elementos que existen, de manera que cada aproximación a las respuestas es un nuevo modo de descubrimiento. Por esta razón, existe quienes argumentan que las "formas científicas" de evaluación nunca harán el trabajo de evaluar las disciplinas artísticas de manera fiable. Dada su ambigüedad, el análisis y evaluación en las disciplinas artísticas son complejos e inclusive, engañosos (Hope \& Wait, 2013).

Asimismo, existe el problema de la "ilusión de totalidad objetiva" respecto de la evaluación cuantitativa. Esta ilusión consiste en pensar que los números y la cuantificación de los aprendizajes sirve para explicar el todo y predecir adecuadamente cada ámbito de cualquier actividad que pueda replicarse usando la fórmula mediante la cual fue generada. Dicha ilusión trae con frecuencia malos usos de la cuantificación y puede dirigir a juicios erróneos sobre lo que ocurre y lo que debe hacerse, particularmente en dinámicas impredecibles como las acciones humanas. Existe mucho del arte que o debe ser replicable ni repetitivo, y, en este sentido, esta ilusión puede perjudicar la 
calidad y logro de las disciplinas artísticas y otros campos (Council of Arts Accrediting Associations [CAAA], 2007).

Expuestas algunas de sus complejidades, vale la pena preguntarse ¿es necesaria la evaluación en carreras artísticas? Entre las razones que hacen pertinente y necesaria la evaluación en carreras artísticas, algunos autores argumentan la necesidad de monitoreo del progreso de sus estudiantes y lo beneficioso de que las instituciones que brindan educación en carreras artísticas puedan dar cuentas sobre sus avances con los mismos (Hoepner, 1984). La evaluación cumple en este sentido, la función de asegurar la calidad y pertinencia de los programas de educación artística, a través de los logros de los estudiantes (CAAA, 2007). En tanto la evaluación es una manera de asegurar información verificable sobre los logros que los estudiantes completarían al terminar un programa de formación, dicha información no solo sirve a la institución sino a los potenciales estudiantes de la misma. El problema de no evaluar los programas artísticos, entonces, es que no se dispondría de información de la efectividad de los mismos para los estudiantes (Wilson, 1968), ¿qué resultados logran luego del programa? ¿Cómo puede comprobarse que los logros de aprendizaje artístico se están cumpliendo sin evaluación?

En educación superior la evaluación informa sobre el inicio, proceso y finalización de los programas de formación. La evaluación es parte de los procesos de admisión y muestra los niveles de conocimientos, habilidades y potencial con los cuales llegan los estudiantes; que deben ser tomados en cuenta en programas de estudios orientados a la creación y performance, incluso para los programas de pregrado. Durante la formación, la evaluación debe -o debería - ayudar a promover y mejorar la calidad del trabajo mientras los artistas más jóvenes y menos experimentados van ganando conocimientos y habilidades. Asimismo, la evaluación demuestra las competencias de los estudiantes para concluir con los programas de formación (CAAA, 2007).

Una vez establecida la importancia de la evaluación se vuelve nuevamente al problema de inicio, ¿qué tan objetiva puede ser la evaluación en disciplinas de las características mencionadas? Existen quienes sostienen que la subjetividad de los criterios de evaluación en las artes desaparece si se plantean como parte del planeamiento del aprendizaje, objetivos educativos emparejados con criterio explícitos y definidos (Gruber, 2008).

Si bien la estandarización en la evaluación es debatible, - y puede serlo aún más en las carreras artísticas-, la ausencia relativa de contenidos y secuencias definidas de instrucción en arte se debe, en parte, a la falta de mecanismos útiles de evaluación del progreso en la formación artística (Hoepner, 1984). La evaluación colaboraría también al progreso de esclarecimiento de los objetivos de los 
programas y por tanto, de su contenido instruccional. Del mismo modo se hace necesario que el público en general y las instituciones educativas en particular tengan claridad sobre qué se está produciendo en la educación artística, y de qué manera se están logrando los desempeños en los estudiantes (McRorie, 1997).

\section{Algunas preguntas en torno a la evaluación artística}

Se parte de que el campo de la evaluación en carreras artísticas es un campo aún en controversia y construcción. En este sentido, existen aún una serie de preguntas a considerar en el estudio de este tema, algunas más generales sobre la educación artística y otras específicas sobre la evaluación en carreras artísticas.

La mirada al currículo en carreras artísticas es inseparable de la mirada sobre su evaluación, por lo tanto, previo a las preguntas sobre evaluación en sí misma, es necesario plantear algunas interrogantes de carácter más general acerca del currículo. Entre ellas, ¿Qué es el conocimiento artístico? ¿Qué cuenta como conocimiento en las artes? ¿Qué aspectos del conocimiento se deben considerar en el currículo de carreras artísticas? ¿Qué aspectos o contenidos son un deber en el currículo de carreras artísticas? ¿A qué se parece un currículo ejemplar en arte? ¿Qué nos dice la investigación sobre prácticas ejemplares en educación artística?

Algunas preguntas generales sobre evaluación en carreas artísticas incluyen: ¿Es válida la evaluación en carreras artísticas? ¿Qué contenidos deben ser evaluados? ¿Existen contenidos que no deben ser evaluados? ¿Qué es lo que se evalúa o debe estar evaluado en la educación artística? ¿Qué califica como criterio ejemplar para la evaluación de carreras artísticas? ¿El proceso de evaluación se contrapone realmente a la creatividad? ¿Qué se busca y se quiere evaluar?

Estas preguntas miran el campo de la evaluación en carreras artísticas desde las cuestiones más generales en relación al currículo y su sentido, hasta cuestiones más específicas como su carácter epistemológico. Estas son planteadas como generadoras para el debate, sin embargo, escapan del objetivo del presente ensayo, que se centra más bien, en responder a interrogantes específicas tales como:

1. ¿Qué es lo que sabemos sobre evaluación en carreras artísticas? ¿Qué se conoce acerca de la evaluación artística en distintos contextos?

2. ¿Con qué propuestas sobre evaluación contamos? ¿Qué aspectos de estas propuestas resultan útiles para la aplicación en nuestro contexto?

Dicho esto, el objetivo del presente ensayo es presentar la información disponible acerca de evaluación en carreras artísticas, que resulte adecuada y pertinente para nuestro contexto (carreras de la Facultad de Arte y de la 
Facultad de Artes Escénicas de la Pontificia Universidad Católica del Perú [PUCP]) y pueda ser usada a favor del mejoramiento de los procesos de evaluación en dicho contexto.

En este ensayo, se toma en cuenta las carreras incluidas en la Facultad de Artes, que se pueden catalogar como de artes plásticas, bellas artes o artes visuales y las de la Facultad de Artes Escénicas (en específico, para las carreas de música, teatro y danza) contempladas en los programas de formación de la PUCP. Se comprende que cada una de las carreras artísticas lidia con diferentes contenidos y formas únicas de comprender al ser humano. Por lo tanto, cada disciplina necesita considerarse en sus propios términos, especialmente cuando el objetivo es la educación de creadores e intérpretes (CAAA, 2007). Si bien se intentará resaltar ciertas particularidades por especialidad, se piensa que hay lugares comunes que agrupan estas carreras afines, por lo tanto, también se discutirá a partir de las generalidades de cada carrera.

El presente ensayo muestra, en primer lugar, una breve revisión sobre historia de evaluación y la evaluación artística. En segundo lugar, luego de una sucinta revisión sobre aspectos generales acerca de la evaluación de los aprendizajes, se presentan las tendencias encontradas para la evaluación en carreras artísticas y algunas propuestas que ejemplifican estas tendencias. Asimismo, se incluye una sección sobre estándares y logros nacionales definidos desde el Council of Arts Accrediting Associations. Finalmente, se hace una integración de una selección de los temas relevantes y se ofrecen recomendaciones para la evaluación en carreras artísticas en nuestro contexto.

\section{SOBRE LA HISTORIA DE LA EVALUACIÓN EN CARRERAS ARTístiCAS}

La evaluación en el ámbito de las carreras artísticas se origina a partir del uso de pruebas estandarizadas que tenían por objetivo la medición del desarrollo cognitivo, el ajuste psicológico u otras habilidades, a partir del logro en destrezas artísticas (Hoepner, 1984; Gruber, 2008). Este es el caso de la prueba "Dibuja a un hombre" de Goodenough (1926 en Gruber, 2008) que, a partir del dibujo de la figura humana se proponía medir otras variables del desarrollo cognitivo y afectivo de los sujetos. Las pruebas estandarizadas provenían de una tradición de ańos anteriores en que se buscaba la clasificación de sujetos para la toma de decisiones para el ámbito educativo. Por ejemplo, con las pruebas de Binet para la medición de la inteligencia y procesos superiores de pensamiento (Bravo \& Fernández del Valle, 2000). Un siguiente origen de las pruebas estandarizadas se situó en el contexto de la Segunda Guerra Mundial, durante la cual se elaboraron las primeras pruebas colectivas de inteligencia 
para seleccionar soldados adecuados a las distintas tareas requeridas (Bravo \& Fernández del Valle, 2000).

Más adelante, en continuidad con la búsqueda de estandarización, se desarrolló pruebas que determinaban específicamente habilidades artísticas. Entre ellas, el McAdory Art Test, creado en 1929 y el Meier-Seashore Art Judgement Test, del año 1930. Así, las pruebas generadas para evaluar los logros de aprendizaje en arte fueron desarrolladas bajo la luz de la medición de habilidades específicas. La prueba llamada "Test de habilidades fundamentales de las artes visuales" (Lewrenz, 1927 en Hoepner, 1984), contemplaba sub-pruebas que medían producción, percepción estética, e historia del arte en niveles de educación básica. Asimismo se desarrollaron pruebas en arte, diseño y crítica para niveles superiores de educación. Sin embargo, muchas de estas fueron construidas a partir de constructos de aptitudes artísticas definidas con poca rigurosidad (Hoepner, 1984).

En Estados Unidos, los libros de texto en el campo de la educación artística mencionaban poco acerca de evaluación hasta antes de 1960. Esto puede haberse debido a la poca consideración de los cursos artísticos como disciplinas académicas, y a la creencia de que estos no requerían las mismas prácticas sobre enseñanza, ni por consiguiente sobre evaluación. Sin embargo a finales de los años 60, comenzó en Estados Unidos el boom del rigor y la medición en el ámbito educativo, con un énfasis especial en la rendición de cuentas (Gruber, 2008). En este momento empezaron las menciones en la literatura sobre la evaluación de las disciplinas artísticas en la educación básica; para luego, a mediados de los años 80 , terminar por considerar la educación en arte una disciplina académica distinguida (Efland, 1990 en Gruber, 2008). No obstante, para ese entonces la evaluación en aula no es aún sistemática, ni popular, y la investigación en evaluación es aún escasa. Al respecto, Boughton (1997) encuentra que entre los años 1959 y 1974, en los artículos escritos en la revista "Studies in Art Education", solo un 5 \% de los artículos escritos tenían que ver con la evaluación y la gran mayoría de estos trataban la evaluación de programas educativos, y no la evaluación de los logros de los estudiantes.

Más recientemente, la atención sobre la rendición de cuentas y las tendencias académicas sobre los estándares de aprendizaje junto con las nuevas demandas por clasificar y seleccionar estudiantes de una manera estandarizada, se han combinado para crear un interés del sistema educativo por estrategias para la evaluación (Gruber, 2008; Bravo \& Fernández del Valle, 2000). La literatura en evaluación, a partir de 1974 se ha incrementado, sin embargo, la razón principal parece justamente responder a la urgencia de imperativos políticos que necesitan información para demostrar y rendir cuentas sobre los fondos invertidos en educación artística para la educación básica (Boughton, 1997). 


\section{Sobre La DEFINICIÓN Y ObJetivos de La EVALUACIÓN EN EDUCACIÓN SUPERIOR}

En su libro Manual de evaluación educativa, Casanova (1999) explica la evolución del concepto de evaluación a lo largo del tiempo. En un primer momento, los modelos de evaluación preponderantes concebían a la evaluación como "el proceso que permite determinar en qué grados han sido alcanzados los objetivos educativos propuestos" (Tyler, 1950 en Casanova, 1999). Así, la evaluación era comprendida exclusivamente como comprobación de resultados educativos. Más adelante, fue definida como el recojo sistemático y uso de la información para tomar decisiones sobre un programa educativo (Cronbach, 1963 en Casanova 1999). De esta forma, la evaluación no es considerada solo como una valoración determinada de los resultados educativos sin afán de intervención, sino que se considera como retroalimentación orientada a la toma de decisiones. En un siguiente momento, se incluye en la evaluación un componente de valoración, que integra la validez y el mérito de lo que se realiza para la toma de decisiones. Así, se incorpora la consideración de la ideología del evaluador y el sistema de valores de la sociedad que van a condicionar la forma en la que se lleva a cabo la evaluación y, en cierta forma, los resultados obtenidos.

De esta forma, Casanova (1999) define la evaluación aplicada a la enseñanza y aprendizaje como:

[...] un proceso sistemático y riguroso de recojo de datos, incorporado al proceso educativo desde su comienzo, de manera que sea posible disponer de información continua y significativa para conocer la situación, formar juicios de valor con respecto a ella y tomar decisiones adecuadas para proseguir la actividad educativa mejorándola progresivamente (p. 60)

Álvarez (2001) agrega que la evaluación en el ámbito educativo es una actividad crítica del proceso de enseñanza - aprendizaje, pues se asume que es una herramienta para adquirir conocimientos. En este sentido, la evaluación actúa al servicio del conocimiento, del aprendizaje y de los intereses formativos a los que debería apuntar.

Así, la evaluación es tanto proceso como resultado (Escobar, 2007), implica la valoración de estos (González, 2001) y la toma de decisiones sobre esta valoración. Comprende la búsqueda y obtención de información de diversas fuentes acerca de la calidad del desempeño, avance, rendimiento o logro del estudiante, y de la calidad de los procesos empleados por el docente, la determinación de su importancia y pertinencia de conformidad con los objetivos de formación (Ianfrancesco 2002 en Escobar, 2007). 
Por otro lado, la calidad educativa puede entenderse desde distintas aproximaciones. Aquella que viene indiscutiblemente ligada a la evaluación es la calidad educativa comprendida como transformación y cambio (Cano, 1998). Esta concepción está vinculada, por un lado, a la mejora del aprendizaje y rendimiento de los alumnos, y, por otro, a la mejora a nivel institucional. Sin embargo, la calidad desde esta perspectiva corre el riesgo de centrarse en la mejora de los resultados académicos (Tiana, 1993 en Cano, 1998).

Adicionalmente, Álvarez (2001), al resaltar algunas características fundamentales de la evaluación, señala que esta debe:

1. Ser democrática. Debería asegurar la participación de todos los afectados por ella, especialmente alumnos y docentes, quienes no serían receptores pasivos o espectadores, sino agente activos para la toma de decisiones sobre su proceso evaluativo.

2. Estar al servicio de los protagonistas del proceso de enseńanza y aprendizaje (docentes y estudiantes). En este sentido, debe estar al servicio de la práctica para mejorarla, y de los sujetos, para beneficiarse de ella.

3. Ser un ejercicio transparente. Los criterios de valoración han de ser explícitos, públicos, y publicitados y negociados entre el docente y los estudiantes.

4. Estar integrada al currículo; estar compuesta por tareas conectadas de manera que sea procesal, continua e integrada. Esto quiere decir que se debe evaluar durante todo el proceso de aprendizaje para dar tiempo a mejoras y ajustes, tanto desde los docentes como desde los estudiantes.

5. Ser formativa, motivadora y orientadora, no sancionadora. En este sentido, se aleja de la mera clasificación de los sujetos y se acerca más a su potencial educador.

6. Ser triangulada. Debería ser ejecutada además de por el profesor, en espacios de coevaluación y autoevaluación.

7. Asegurar la calidad de los aprendizajes.

Por su parte, Bravo y Fernández del Valle (2000) exponen la aparición de ciertas demandas no cubiertas por la evaluación convencional y las pruebas estandarizadas. Estas demandas vienen siendo respondidas con nuevas tendencias en evaluación como la evaluación auténtica, que, según estos autores, implica que la persona produzca o genere un objeto mediante un proceso que tenga la duración suficiente para poder ser evaluado junto con su resultado. Desde este modelo se busca reivindicar la importancia del contexto, el realismo de las demandas de la situación de instrucción y un mayor protagonismo 
del proceso frente a los resultados. Como afirma Messick (1998 en Bravo \& Fernández del Valle, 2000).

[...] la tarea debe dejar libertad al examinado; el material estimular no debe estar estandarizado; han de ser las propias personas evaluadas las que elijan el momento de actuar para estar verdaderamente motivados; incluso, y especialmente en el ámbito educativo, el evaluador debe conocer a las personas que está evaluando, sus circunstancias vitales y su historia de ejecución de la tarea (p. 97).

Las definiciones y concepciones de evaluación revisadas en este apartado sirven como marco de referencia para comprender la evaluación de los aprendizajes. Sin embargo, si bien son útiles en la comprensión de la evaluación en carreras artísticas y una guía para comenzar el trabajo y comprender el sentido de la evaluación en general, quedan aún en un plano que no permite comprender con la especificidad requerida los lineamientos para la evaluación de las disciplinas artísticas. Por este motivo, en la siguiente sección, se exponen propuestas específicas de evaluación para el caso de los aprendizajes artísticos.

\section{Algunas propuestas para eValuar en Carreras artísticas}

En la búsqueda de propuestas de evaluación específicas para el campo de las carreras artísticas se ha identificado dos tendencias, principalmente. Por un lado, están aquellos autores que se acercan y valoran la estandarización de las pruebas artísticas (con un rango de flexibilidad desde lo más flexible hasta lo más rígido en cuanto a evaluación por criterios) y, por otro, aquellos que más bien se oponen a la estandarización y a la generación de criterios de evaluación desde fuera de la comunidad educativa. Debe recalcarse que estas propuestas se basan en la investigación para la educación básica, pero son aplicables en la educación superior.

En la primera tendencia, se encuentran autores enfocados en el establecimiento de mejores objetivos, criterios y estándares para medir los aprendizajes en arte, que sean más adecuados y se acomoden mejor al tipo/cualidad de los aprendizajes en arte, algunos con el objetivo final de la evaluación - $\mathrm{y}$ certificación de la calidad - de programas de formación artística y otros, con un afán de mejora en la medición de los logros para la rendición de cuentas (Wilson, 1968; Hoepner, 1984; Kay Beattie, 1997; Ramsay, 2005; Gruber, 2008; DeLuca, 2010). Así, esta tendencia relaciona la evaluación de programas —una mirada macro—, con la evaluación de aprendizajes — mirada micro—, para la búsqueda de la calidad. 
Dentro de la segunda tendencia, están quienes critican estas formas estandarizadas de evaluación, con más o menos alejamiento del establecimiento de criterios, estándares y objetivos: por un lado, se propone una forma inductiva de establecimiento de criterios de evaluación, en la que comunidad establece y delimita sus propios criterios y estándares, consistentes con las prácticas de comunidades similares y coherentes con la naturaleza dinámica de la disciplina artística (Boughton, 1997). Por otro lado, se recogen y teorizan experiencias de evaluación de los aprendizajes por docentes de Música, alejados de la estandarización que buscan un proceso que contemple la creatividad de los estudiantes y sus niveles de dominio o maestría de las técnicas aprendidas (Hope \& Wait, 2013).

En el siguiente acápite se resumen algunas de las propuestas mencionadas para cada tendencia. Finalmente, se presenta una sección con los lineamientos para la evaluación elaborados por el Council of Arts Accrediting Associations (CAAA, 2007), para sus propuestas de estándares, objetivos y criterios para la acreditación de programas de formación artística.

\subsection{Evaluar con criterios: las propuestas de Donna Kay Beattie (1997) y Christopher DeLuca (2010)}

\subsubsection{Criterios, objetivos y estándares en la evaluación en carreras de artes visuales - Donna Kay Beattie (1997)}

La propuesta de Beattie (1997) defiende la existencia de estándares y criterios explícitos para la enseñanza de las artes. Como razones para sustentar su propuesta, sostiene que (a) los criterios y estándares explícitos son beneficiosos para comprender y explicar los resultados de los estudiantes; (b) los estándares explícitos funcionan de manera más objetiva: los objetivos son más claros y las evaluaciones menos engañosas para los estudiantes; (c) los estudiantes terminan siendo juzgados por los estándares y no por los docentes, ya que, cuando son explícitos, se vuelven externos y tanto profesores como estudiantes trabajan colaborativamente para lograrlos; (d) Tener criterios y estándares elaborados hace más sencilla la tarea de evaluación al docente que no tiene mucha experiencia evaluando, de la forma más objetiva que pueda lograrse; (e) los estándares a nivel nacional facilitan la comparación del desempeño entre instituciones; (f) los estándares a nivel nacional permiten la movilidad de los estudiantes entre instituciones; y (g) los estándares a nivel nacional son efectivos para las instituciones, ya que aquellas que cuentan con estándares hechos para el nivel nacional no tienen que invertir tiempo y recursos en elaborar los propios. 
Antes de entrar a la propuesta de Beattie, es importante comprender las definiciones que ella usa para comprender los estándares, los criterios y los objetivos. En primer lugar, la autora define estándar como "un nivel definitivo de excelencia o un grado definitivo de calidad, visto como un producto del esfuerzo o una medida reconocida de que es adecuado para cierto propósito, establecido así por una autoridad, costumbre o consenso". Sandler (1986 en Beattie, 1997) afirma que un estándar es siempre una abstracción y nunca un objeto. De este modo, el estándar es un punto de referencia consensuado, relativo a un criterio, que requiere un juicio que determine cuán bien la conducta se acerca al ideal esperado (Boughton, 1996 en Beattie, 1997).

En segundo lugar, un criterio se define como "una propiedad, característica o dimensión de cualquier objeto mediante la cual su calidad puede ser juzgada o estimada o mediante la cual una decisión o clasificación puede llevarse a cabo" (Sandler, 1986b en Beattie, 1997). Los criterios son jerárquicos y, por lo tanto, en los sistemas de evaluación por criterios, estos necesitan estar conceptualmente organizados, y esquemática y prácticamente ordenados para registrar los logros de los estudiantes (Sandler, 1986c en Beattie, 1997). De esta forma, los criterios pueden existir sin estándares, pero los estándares no existen sin criterios. Ejemplos de criterios para las habilidades de creación artística que propone Beattie son a) concepto, b) forma, c) técnica, d) medio, e) presentación. Los subcriterios para a) concepto, pueden incluir a.1) fuerza, a.2) relevancia, a.3) originalidad, a.4) contexto, a.5) investigación, a.6) cómo se traduce en forma, etc. Para Beattie, los criterios más grandes o generales son adecuados para la evaluación formativa y la retroalimentación en clase. Los subcriterios más específicos son mejores para la evaluación sumativa y de gran escala. Asimismo, existen criterios "temporales", aquellos que se diseńan dentro de la institución y están en continua evaluación y replanteamiento, como en la evaluación formativa; y criterios "de salida", que determinan los niveles de logro y generalmente se asocian a evaluación sumativa o de salida.

Finalmente, se entiende por objetivo "aquello que los estudiantes deben ser capaces de saber (hacer), valorar, o sentir al concluir un segmento de instrucción" (Nitko, 1996 en Beattie, 1997). Los objetivos son potenciales pues indican aquello que se intenta lograr y de esta forma sirven como organizadores de las experiencias de aprendizaje (Sandler, 1987b en Beattie, 1997). Están basados en las dimensiones destacadas de una disciplina o asignatura, y se relacionan con el contenido, procesos, habilidades, y afectos. Los criterios de un curso se encarnan en los objetivos (fines, metas u objetivos generales).

Una vez expuestas las definiciones que Beattie utiliza para comprender los estándares, criterios y objetivos, se puede pasar a revisar su propuesta. 
La autora plantea que se definan criterios a nivel nacional para que cada programa artístico se cree en relación con los mismos, conservando su carácter único y distintivo. No propone estándares a nivel nacional para juzgar los aprendizajes de los estudiantes, ya que estos estarían muy lejanos de cada contexto educativo particular, pero sí sostiene que los criterios que generan los marcos de trabajo deben establecerse, para luego poder construir programas en educación artística contextualizados para cada institución. La evaluación en este sentido debe ser interna (para cada institución) pero también externa (para el nivel nacional).

Para la definición de criterios, Beattie defiende un proceso colaborativo, en que tanto estudiantes como profesores determinan los criterios e indicadores de logro. Sostiene que los estudiantes deben conocer anticipadamente los criterios que organizan la evaluación y con los cuales su trabajo será juzgado. Según la autora, los estudiantes ganan considerablemente en conocimiento cuando se les enseńa a generar su propio conjunto de estándares.

Por otro lado, los estándares deberían poder incorporar los cambios en la dificultad de la tarea, la comprensión y variación en la comprensión de los criterios, y los cambios en las performances de los estudiantes. Incluso con los estándares explícitos, los docentes deben tener un margen de libertad para aplicar e interpretar los criterios, y para organizarlos en relación a los desempeños de los estudiantes.

La idea de Beattie es que los estándares no sean tan explícitos (concretos, estructurados, definidos) como para ser demasiado rígidos, ni tan flexibles o poco estructurados como para que no puedan ser comprendidos y ejecutados. Estos son construcciones sociales antes que verdades objetivas; son juicios de valor, establecidos en un contexto particular, para una demanda social relativa al mismo. Los estándares deben ser creados antes que los trabajos de los estudiantes, para que estos puedan monitorear su propio progreso en el camino de conseguir los objetivos.

Por supuesto, la propuesta de Beattie funciona para el contexto de Estados Unidos, donde ya se han establecido estándares nacionales, con el objetivo de la acreditación de carreras artísticas (CAAA, 2012 en Hope \& Wait, 2013). Esta es una propuesta que compromete particularmente al Estado. Se debe comprender esta propuesta en función de la acreditación y rendición de cuentas: intenta demostrar la existencia de procedimientos que pueden asegurar la calidad de la comparación entre instituciones. 


\subsubsection{La capacidad de evaluación en la educación artística-Christopher DeLuca (2010)}

DeLuca (2010) explica los conceptos de la evaluación del aprendizaje (EDA) y de la evaluación para el aprendizaje (EPA), para proponer la EPA como una estrategia en la evaluación de aprendizajes de disciplinas artísticas. Define la EPA como aquella evaluación básicamente sumativa, que implica generalmente pruebas de lápiz y papel, y alternativamente otros métodos de evaluación que incluyen rúbricas, portafolios y evaluación de procedimientos. Por otro lado, para el autor, la EPA consiste de evaluación formativa, aquella evaluación que ocurre entre tareas sumativas que promueven el aprendizaje. Este tipo de evaluación difiere de la evaluación formativa común, en que involucra activamente a los estudiantes en su evaluación, mediante la coevaluación y la autoevaluación.

Los cinco principios que caracterizan a la EPA son:

1. Los profesores dan retroalimentación efectiva a sus estudiantes.

2. Se promueve el involucramiento activo de los estudiantes en su evaluación, mediante la autoevaluación.

3. La retroalimentación por parte de compañeros, profesores y de sí mismos como estudiantes provee guías para la enseñanza y el aprendizaje.

4. Se reconoce que la evaluación tiene una profunda influencia en la motivación de los estudiantes.

5. La motivación es un factor clave para la comprensión de los estudiantes de sus procesos de aprendizaje. (Assessment Reform Group, 1999 en DeLuca, 2010)

En la EPA la evaluación, la enseñanza y el aprendizaje se conjugan para mejorar los logros, el desarrollo de la metacognición, los aprendizajes motivados y las percepciones positivas de los propios estudiantes a través de las actividades de evaluación. La idea es que los estudiantes puedan evaluar sus propias habilidades y desarrollar un plan para conseguir las metas de aprendizaje propuestas. Además, a través de la coevaluación, se genera una comunidad/ cultura de aprendizaje en la que la reflexión se hace en conjunto y se vuelve un apoyo para el desarrollo de aprendizajes.

Dicho esto, la propuesta del autor es usar la EPA en la evaluación de las artes. Debido a que este tipo de evaluación promueve las habilidades fundamentales necesarias para el desarrollo de la creatividad, como, por ejemplo, la reflexión y autoevaluación de los procesos de aprendizaje. Estas habilidades ayudan a los artistas a lograr mejores productos y promover su capacidad creativa. 
Asimismo, la EPA es una aproximación que facilita la creación de comunidades de aprendizaje: así como los artistas profesionales construyen comunidades que responden y retroalimentan su producción artística, los estudiantes, mediante esta aproximación, inician procesos de crítica y valoración sobre sus productos. Así, esta aproximación desarrolla al estudiante como artista y genera legitimidad para la colaboración en la evaluación crítica. Más aún, contribuye con la apreciación de la complejidad de las propuestas artísticas.

Además, esta propuesta implica que los criterios de evaluación formativa promuevan la creatividad en vez de limitarla. Se busca que estos sean lo suficientemente abarcadores para adaptarse a la flexibilidad de los procesos y productos artísticos. De esta forma, el autor propone rúbricas como ejemplos de calificación de criterios flexibles de evaluación que guíen al estudiante sobre cómo mejorar sus logros promoviendo la creatividad. Los criterios flexibles que propone DeLuca se basan en la novedad de los productos o piezas de arte producidas por los estudiantes, y consideran cómo se han conectado distintas técnicas para representar un tema, cómo se ha contextualizado el producto a favor de su interés visual y de qué manera se presenta para que su significado alcance niveles que superen lo esperado. El criterio promueve que los estudiantes piensen cómo generar abstracciones que provoquen entendimientos más profundos en su propuesta artística, en lugar de definir cómo debería verse el producto final.

\subsection{Reconsiderando la estandarización: las propuestas de Doug Boughton (1997) y Hope y Wait (2013)}

\subsubsection{Reconsiderando la evaluación y los estándares de logro en la educación artística - Doug Boughton (1997)}

Boughton (1997) sostiene que los estándares nacionales generados en Estados Unidos para los logros en educación artística no muestran los logros del trabajo de los estudiantes de manera consistente y significativa. Por este motivo, propone alternativamente a "la comunidad como árbitro de la calidad" a manera de estrategia de evaluación para la delimitación de estándares de juicio consistentes con la práctica de otras comunidades y más coherentes con la naturaleza dinámica de las disciplinas artísticas.

Para Boughton los estándares son comprendidos como el nivel determinado de desempeño esperado, en relación a un ideal o ejemplar de desempeño. El problema con medir el desempeño en habilidades artísticas, según el autor, es que no existe una conducta ejemplar o ideal con la cual compararlo. La tarea es más compleja porque en las artes visuales, particularmente en la generación de obras artísticas, los productos deben mostrar un grado de originalidad 
en su concepción y cierto sentido del carácter idiosincrático de su creador. Por lo tanto, afirma que es bastante inapropiado tratar de establecer un ideal de este desempeńo o del producto fruto del mismo.

Asimismo, Boughton establece que los criterios no proveen más que lineamientos para la evaluación. Según el autor, los criterios de evaluación de objetos artísticos suelen ser incapaces de mostrar la ambigüedad de las cualidades visuales y sus significados, aspectos especialmente difíciles de comprender por personas con poca experiencia en este rubro. De esta forma, los criterios pueden ofrecer ventanas conceptuales que guíen la mirada del jurado frente a una obra, pero no proveer un marco definitivo para juzgar la calidad de todos los trabajos por igual. Los criterios pueden expresar cualidades deseables que pueden o no ser relevantes para una pieza de arte o una colección de piezas, pero queda del observador comprender el género de la pieza y su contexto de producción, y decidir el conjunto de criterios y la valoración mediante los cuales juzgarla.

En el mundo del arte y la comunidad de artistas, la calidad y valor de las nuevas expresiones artísticas se debate y critica hasta que se llega al consenso. Boughton argumenta que si se quiere ser coherente con esta tendencia de la comunidad, en el ámbito de la educación artística, también debería tenerse este debate al analizar los productos de los estudiantes. Así, Boughton propone que, en vez de guiarse por estándares ya establecidos en documentos de alcance nacional, se empleen procesos evaluativos basados en la noción de comunidades como árbitros de calidad para determinar estándares adecuados para la evaluación de la educación artística. Este procedimiento es consistente con las prácticas de la comunidad de artistas en general, y consecuente con la naturaleza dinámica de las artes.

El proceso que describe Boughton comienza con el planteamiento de referentes de logro. Esto puede variar bastante en cada contexto, pero, por lo general, se pueden seleccionar trabajos de una población representativa de estudiantes, y contrastarlos con criterios acordados a priori. La intención es representar el rango de posibilidades de logros potenciales que puede establecerse a partir de un conjunto de trabajos. Este proceso puede tomar varios días, en los que se debate intensamente hasta que el rango de criterios es establecido por consenso. Los criterios y objetivos, entonces, son interpretados en el contexto de trabajos reales que representan los estándares acordados. Estos constituyen parte del conocimiento compartido de los expertos y son relativamente inaccesibles (poco comprendidos) por personas con poca experiencia en las artes. El conocimiento tácito de los jueces experimentados es transmitido a los jueces novatos mediante la participación conjunta en la actividad evaluativa. La dificultad particular es que un estándar debería verse representado en trabajos diferentes. 
Luego de haber planteado referentes de logro, se pasa a un "proceso de moderación" en el que comparan los trabajos de los estudiantes contra los referentes de logro, en un espacio de debate crítico y búsqueda de consenso.

\subsubsection{Evaluación en nuestros propios términos - Hope y Wait (2013)}

Hope y Wait (2013) proponen alejarse por completo de las evaluaciones estandarizadas y generar un proceso que contemple la creatividad de los estudiantes y sus niveles de dominio o maestría de las técnicas. La propuesta parte de un intento por explicar los principios artísticos que rodean a su disciplina, la música, así como los acercamientos y filosofía usada para evaluar su trabajo.

Para estos autores, la naturaleza de la evaluación exitosa en temas artísticos depende de la comprensión profunda de los objetivos del creador y de ser capaz de evaluar su éxito en conseguirlos. Ya que existe un número infinito de posibilidades de logros, y ya que las decisiones sobre las mismas están hechas por individuos, las evaluaciones efectivas requieren conocimiento profundo y un alto grado de sofisticación. Por esta razón, los autores defienden la evaluación individual en vez de la estandarizada.

Ocurre en evaluación que, en vez de observar el todo, se establecen criterios que desagregan los objetos en sus partes: según los autores, en evaluación es necesario considerar los "todos" y no las partes o elementos. Si bien sí se pueden evaluar estas, lo más importante es entender cómo trabajan juntas para producir un resultado de objeto o composición.

Acerca de la evaluación en "técnica", los autores sostienen que sí se puede evaluar el progreso hasta el dominio completo de la técnica en novatos, pero esto cambia para individuos con cierta profesionalización. Lo que plantean es que la evaluación del progreso debe necesariamente ser compleja. No se trata de respuestas dicotómicas sobre la obtención de un grado de dominio de la técnica, sino que se involucre el dominio de la técnica junto con las decisiones estéticas del artista sobre su uso. La precisión no es equivalente suficiente a la calidad.

En la música, se tienen marcos estructurales (diferentes formas de composición musical existentes son ejemplo de estos marcos) y sistemas (desarrollados por músicos y por la misma práctica musical) que se desarrollan, evolucionan y generan nuevos estándares que se integran con las diversas técnicas musicales.

Consistente con lo dicho anteriormente, la evaluación exitosa y efectiva depende de un sofisticado grado de comprensión de la integración de los marcos, sistemas y medios técnicos para producir un "todo" específico: ya sea una performance, un acto de enseñanza, un trabajo, etc. 
En la propuesta de evaluación según Hope y Wait, los individuos interpretan para sus maestros y otros invitados que conforman jurados diversos. Las interpretaciones se siguen de críticas del público, de pares. Se tiene un amplio rango de muestras: algunas son públicas pero muchas otras internas, y se compite por papeles y lugares en ensambles. Se trabaja mucho en audiciones y revisión de portafolios. Asimismo, se tiene asesoría constante durante ensayos y lecciones particulares. Por supuesto, se busca asesoría y crítica de otros artistas y particularmente, se busca la comparación en habilidades técnicas con ellos. Adicional a estas formas de evaluación específicas para el campo, se tienen otras que podrían considerarse comunes a otras áreas, tales como evaluación del trabajo por asignaturas, evaluación de proyectos, pruebas de logros y aptitudes, etc.

\subsection{Propuesta del Consejo de Asociaciones de Acreditación de las Artes (Council of Arts Accrediting Associations, 2007)}

En Estados Unidos, El Consejo de Asociaciones de Acreditación de las Artes brinda lineamientos sobre la evaluación de logro de los estudiantes en disciplinas artísticas. A continuación, se presentan algunas de las ideas principales encontradas en estos lineamientos.

En la propuesta de la CAAA, se considera que la naturaleza del arte define cómo considerar logros y calidad en términos de las formas de arte en sí misma. Así, se provee una serie de características a las instituciones para que estas puedan considerar cómo definir calidad en sus propios términos, así como lo hacen con otras disciplinas no artísticas. Considerar una disciplina en sus propios términos significa trabajar en su evaluación en términos de su intención, contenidos, procesos y productos. La CAAA afirma que las disciplinas artísticas usan una amplia variedad de mecanismos de evaluación "históricamente-probados". Algunos de ellos implican crítica de otras personas. La evaluación en carreras artísticas es sumativa y formativa. Los mecanismos incluyen audiciones, portafolios, exámenes por cursos y por jurados, clases maestras, performances, clases técnicas o de creación, ensayos, exhibiciones, performances y muestras abiertas al público.

La propuesta de estos mecanismos no está basada en métodos cuantitativos de evaluación. Sin embargo, existen ciertos aspectos del dominio de la disciplina que tienen respuestas cerradas, o que se basan en profesionalismo técnico y necesitan de la constancia y repetición. Muchos de estos aspectos sí pueden ser evaluados efectiva y honestamente de una forma cuantitativa. De cualquier manera, los números, por más vitales y útiles que sean, no pueden producir o reproducir las piezas de arte, ni abarcarlas, ni siquiera a nivel de las tecnologías 
que pueden reproducir ciertas formas artísticas. Incluso para los ejemplos digitales, los números son los medios del arte, no el arte en sí mismo.

Por otro lado, las explicaciones verbales o escritas preparadas para la evaluación de un producto son distintas del logro del mismo. Las habilidades para hablar, escribir y comunicar son importantes, pero es mucho más importante que la visión del artista sea evidente a través de su trabajo que tener un enunciado u afirmación en palabras.

\section{INTEGRACIÓN Y RECOMENDACIONES}

A continuación, se ofrecen, a manera de resumen y reflexión acerca de los temas sobresalientes de las distintas propuestas, algunas recomendaciones aplicables a la evaluación de las carreras artísticas en general, tanto en artes visuales, como en artes escénicas.

\subsection{Sobre la estandarización}

Se puede observar que las propuestas que contemplan la generación de estándares a nivel nacional se enfocan en sus beneficios para la comparación entre instituciones, con el objetivo de la acreditación de instituciones y la rendición de cuentas.

La evaluación estandarizada basada en comparaciones nacionales, regionales o provinciales es una responsabilidad del Estado y, por tanto, escapa a la responsabilidad de la universidad. Sin embargo, habría que pensar de qué manera los procesos que se implementen a nivel institucional para asegurar la calidad de los programas de formación y brindar información sobre la misma a estudiantes y postulantes puede impactar en el desarrollo a largo plazo de estándares como los propuestos. Es necesario, en este sentido, establecer el rol de las instituciones para el establecimiento de estándares que sirvan como referente para políticas de mayor alcance.

Como menciona Hoepner (1984), la estandarización de la evaluación generará programas de estudio y contenidos más estandarizados. En consecuencia, el debate sobre los beneficios de la estandarización a nivel nacional también involucra el debate sobre calidad de los programas formativos y la reflexión sobre la necesidad de la estandarización de esos programas.

\subsection{Sobre el desarrollo de objetivos, estándares y criterios}

Destaca la importancia de establecer objetivos de logro, estándares y criterios que guíen la evaluación de los aprendizajes de los estudiantes. Los objetivos, 
estándares y criterios deben ser parte del plan de formación y, por lo tanto, del plan de evaluación de las facultades, en miras, por ejemplo, a la acreditación de carreras artísticas.

Es deseable que los objetivos sean claros y pertinentes, y expresados a manera de conductas observables. Se espera que brinden información sobre las habilidades de los estudiantes y que admitan que estas sobrepasen las expectativas de logro. A partir de estos objetivos, se deberían generar los estándares de progreso de los estudiantes.

Los criterios que miden estos objetivos, por su parte, deben ser flexibles y capturar las cualidades únicas y genuinas de los productos artísticos y de la propuesta de cada estudiante. En lugar de indicar cómo deben verse los productos, deben promover la creatividad en las propuestas. Sin embargo, se espera demostrar mediante el consenso que las interpretaciones y propuestas responden a los criterios propuestos, y establecer ejemplos o evidencia del trabajo de los estudiantes que fundamenten los criterios establecidos.

El establecimiento de criterios debería ser un proceso colaborativo, donde participen profesores, estudiantes y autoridades. Además de la colaboración en el establecimiento de criterios, es favorable promover que los estudiantes generen sus propios indicadores de logro.

De modo similar, es esperable que los docentes tengan un margen de libertad en la aplicación e interpretación de los criterios, para su organización en relación a las actividades propuestas y a los desempeńos de los estudiantes. Los profesores deberían planear su evaluación en base a los criterios generados colaborativamente.

\subsection{Sobre la información para estudiantes}

Los estudiantes deben contar con información sobre los objetivos de la evaluación y los mecanismos y actividades de evaluación que van a ser utilizadas. La intención de la evaluación debe estar conectada a los resultados de aprendizaje de los distintos cursos y las disciplinas.

Asimismo, los estudiantes deben conocer los estándares y criterios esperados, y se espera que formen parte del proceso de elaboración de los mismos.

\subsection{La aproximación a la evaluación}

La aproximación a la evaluación de los aprendizajes debe considerar ser formativa y sumativa. Esta debe comprender la retroalimentación constante por parte de profesores, pero también de los propios estudiantes en el involucramiento activo en la evaluación, mediante la auto y coevaluación. Tal como lo hacen los 
artistas profesionales, los estudiantes deben verse influenciados por la crítica y valoración de sus productos y propuestas por parte de sus compañeros.

Se debe otorgar espacio para la discusión, reflexión y autorreflexión sobre los trabajos de los estudiantes. Esta debería orientarse por los objetivos y criterios que persiguen las disciplinas y también por los cánones de apreciación artística. Se espera que la valoración sea objetiva dentro de estos parámetros.

\subsection{Uso de estrategias integradas}

La evaluación en disciplinas artísticas debe contemplar la mayor variedad de estrategias para evaluar. Asimismo, se debe evaluar productos artísticos diversos. Las estrategias deben ajustarse a los productos evaluados e incluir la observación como un insumo adicional a la evaluación formativa en la creación artística.

La evaluación de productos debe tomar en cuenta y establecer un balance adecuado entre cuánto de la evaluación está midiendo el talento previo del estudiante, cuánto habilidades desarrolladas previamente a los cursos y cuánto la educación recibida.

Adicionalmente, es importante tomar en cuenta el nivel de avance en la carrera del estudiante y plantear estrategias que contemplen esta diversidad. Para los principiantes, la evaluación se dirigiría más al dominio de la técnica, y para los avanzados, a aspectos que abarcan una mayor exigencia, como, por ejemplo, las decisiones estéticas del artista. La evaluación debe ser necesariamente compleja.

\subsection{Sobre evaluación en nuestro contexto}

Finalmente, es importante mencionar que es poco lo que se sabe sobre cómo se está llevando a cabo la evaluación en las carreras artísticas en nuestra universidad. Se esperaría que la evaluación funcione en base a los objetivos que se han planteado desde cada especialidad y los perfiles de estudiantes y egresados elaborados por cada especialidad de la PUCP. Sin embargo, no es posible confirmarlo sin llevar a cabo investigación empírica que levante información sobre la evaluación en cada especialidad.

Para una mejor precisión de las recomendaciones aquí expuestas, se sugiere investigar las prácticas mediante las cuales se está llevando a cabo la evaluación en las distintas disciplinas artísticas para elaborar recomendaciones específicas por especialidades, niveles y contemplando las particularidades de cada grupo de individuos. Es importante también notar que a pesar de ser deseables los estándares a nivel nacional, es necesario iniciar con una agenda particular para que esta universidad, desde sus propias unidades, establezca aquello que es necesario priorizar como agenda de políticas pedagógicas internas. 


\section{REFERENCIAS BIBLIOGRÁFICAS}

Álvarez Méndez, J. M. (2001). Evaluar para conocer, examinar para excluir. Madrid, España: Morata.

Beattie, D. K. (1997). Visual arts criteria, objectives and standards: a revisit. Studies in Art Education, 38(4), 217-231. https://doi.org/10.2307/1320522

Boughton, D. (1997). Reconsidering Issues of Assessment and Achievement Standards in Art Education: NAEA "Studies" Lecture. Studies in Art Education, 38(4), 199-213. https://doi.org/10.2307/1320520

Bravo, A. \& Fernández del Valle, J. (2000). La evaluación convencional frente a los nuevos modelos de evaluación auténtica. Psicothema, 12(2), 95-99.

Cano García, E. (1998). Evaluación de la calidad educativa. Madrid, España: La Muralla.

Carpenter, B. S. (2006). Editorial: Exemplary Content, Curricula, and Criteria for Assessment in Art Education. Art Education, 59(6), 4-5. https://doi. org/10.1111/j.1096-0031.2006.00103.x

Casanova, M. A. (1999) Manual de evaluación educativa. Madrid, Espańa: La Muralla.

Council of Arts Accrediting Associations [CAAA] (2007). Achievement and Quality: Higher education in the arts. Excecutive Sumary. Recuperado de http://aqresources.arts-accredit.org/index.jsp?page $=$ AchievementAndQuality

DeLuca, C. (2010). The capacity of assessment in arts education. Encounters on Education, 11, 3-12. https://doi.org/10.15572/ENCO2010.01

Escobar, J. (2007). Evaluación de aprendizajes. Un asunto vital en la educación superior. Revista Lasallista de Investigación, 4(2), 50-58.

González, M. (2001). La evaluación del aprendizaje: tendencias y evaluación crítica. Revista Cubana de Educación Media Superior, 15(1), 85-96.

Gruber, D. (2008). Measuring Student Learning in Art Education. Art Education, 61(5), 40-45.

Hoepner, R. (1984). Measuring students' achievement in art. Studies in Art Education, 4, 25-258.

Hope, S. \& Wait, M. (2013). Assessment in our own terms. Arts Education Policy Review, 114, 2-12. https://doi.org/10.1080/10632913.2013.744235

McRorie, S. (1997). On sexing chickens and other judgements. Studies in Art Education, 38(4), 195-196. https://doi.org/10.2307/1320518

Ramsay, W. (2005). Assessment in visual arts. Curriculum Support, 10, 11-12.

Wilson, B. (1968). A Proposal for Systematic Curriculum Evaluation in Art Education. Studies in Art Education, 9(3), 57-68. https://doi. org/10.2307/1319765 